\title{
NEOTRIE VR: NUEVA GEOMETRÍA EN REALIDAD VIRTUAL
}

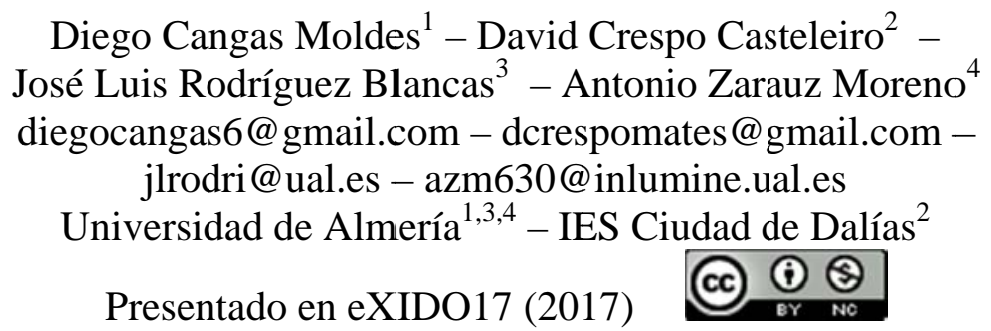

\section{Resumen}

"NeoTrie VR" es un software que permite crear, manipular e interactuar con objetos geométricos y modelos $3 d$ en general, dentro de un escenario de realidad virtual. Su sencillez y facilidad de manejo dota al profesorado de una nueva herramienta de trabajo, que además del estudio de la geometría de manera novedosa, intuitiva y divertida, promueve la creatividad e interacción entre el alumnado.

Palabras clave: Realidad virtual, diseño 3D, HTC Vive, serious game, videojuego, geometría, topología, grafos, poliedros.

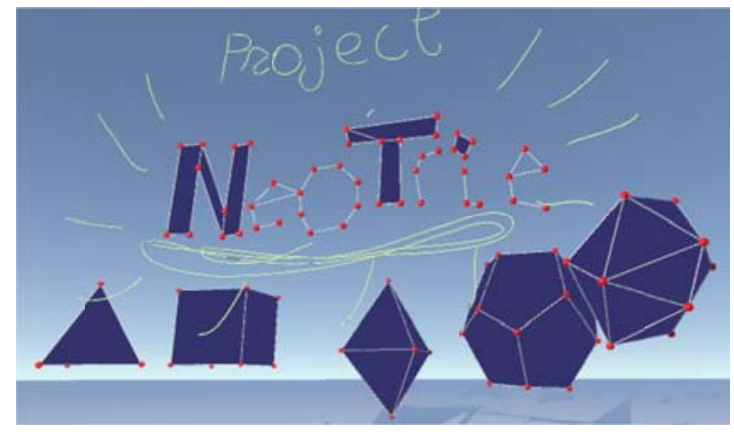

\section{Introducción}

NeoTrie, acrónimo de “Neo” y “Géométrie” del francés, es un "serious game” de realidad virtual que surge del proyecto "¡Juguemos a clasificar superficies!"(1). En octubre de 2016, dicho proyecto recibió el primer premio en la modalidad de Laboratorio de Matemáticas, en la XVII edición del concurso Ciencia en Acción, que se celebró en Algeciras. Está basado en la construcción y estudio de superficies desde un punto de vista topológico. Partiendo de actividades manipulativas concretas, evoluciona hasta el tratamiento de las mismas con medios tecnológicos, como Mathematica, Apps para Android o realidad virtual [VA]. Actualmente, participan más de 70 centros de 17 países [PS].

La primera versión de NeoTrie VR se presentó en el Congreso Bienal de la Real Sociedad Matemática Española, en febrero de 2017, en la Universidad de Zaragoza. Durante el curso pasado y el actual se está utilizado en asignaturas de geometría y 
topología del Grado de Matemáticas de la Universidad de Almería, y se ha testado en una veintena de centros en distintos lugares de España. Destacamos los talleres impartidos durante la exposición Imaginary Arte y Matemáticas, en el museo de Almería, de octubre de 2016 a febrero de 2017, en los que participaron más de 500 alumnos de Enseñanza Secundaria y numeroso público pudo probar el software. En mayo de 2017, se presentó NeoTrie VR también en la 15ª Feria de la Ciencia de Sevilla. En julio de 2017, impartimos una comunicación titulada “Geometría y topología en Realidad Virtual” en las II Jornadas de Experiencias e Innovación Docente en Estadística y Matemáticas, organizadas por la UNED en Úbeda, lo que ha motivado la redacción de este artículo.

Recientemente, en octubre de 2017, NeoTrie VR ha recibido una Mención de Honor en la Modalidad de Matemáticas de la XVIII Edición de Ciencia en Acción, celebrada en Eibar-Ermua.

\section{Objetivos}

Al introducir esta nueva metodología en al aula de Matemáticas, no sólo estamos brindando una ocasión de gamificación al alumno, sino que mediante la misma, favorecemos su proceso de enseñanza y aprendizaje en el que se pretenden alcanzar los siguientes objetivos:

- Familiarizar al alumnado con las Geometrías tanto euclídeas y no euclídeas, así como los elementos básicos de las mismas.

- Favorecer el aprendizaje por descubrimiento e inductivo.

- Educar en la percepción espacial.

- Generar un aprendizaje cooperativo, basado en la dependencia positiva.

- Acercar de un modo lúdico la Geometría y la Topología al alumnado.

\section{Contenidos}

El software que presentamos, complementa a las actividades manipulativas que puedan realizarse en el aula, permite la exposición y estudio de la mayoría de los contenidos que sobre Geometría, desde un punto de vista descriptivo, se realizan en Primaria y Secundaria, y alcanza también temas avanzados que se imparten en la Universidad:

- Elementos euclídeos: puntos, segmento, recta y semirrecta, ángulos, planos, etc.

- Polígonos: Vértices, lados, diagonales y simetrías.

- Poliedros: Vértices, caras, aristas, concavidad y convexidad, planos de simetría 
y fórmula de Euler.

- Simetrías en el plano y en el espacio.

- Superficies topológicas: Género, borde, orientabilidad y fórmula de EulerPoincaré

- Curvas y superficies a partir de sus parametrizaciones.

- Problemas sobre nudos y enlaces.

- Resolución de problemas clásicos de grafos.

- Otros por concretar según evolucione el software.

\section{La realidad virtual}

La realidad virtual es una tecnología en auge que permite introducir al usuario dentro de mundos virtuales generados totalmente por ordenador. Sus comienzos, hay que situarlos en la década de los años 90, aunque las expectativas suscitadas estuvieron por debajo de la aceptación del gran público. El máximo promotor en la década actual fue Palmer Lucker, con su ya conocido Oculus Rift, llegando incluso a encandilar a Facebook, que lo

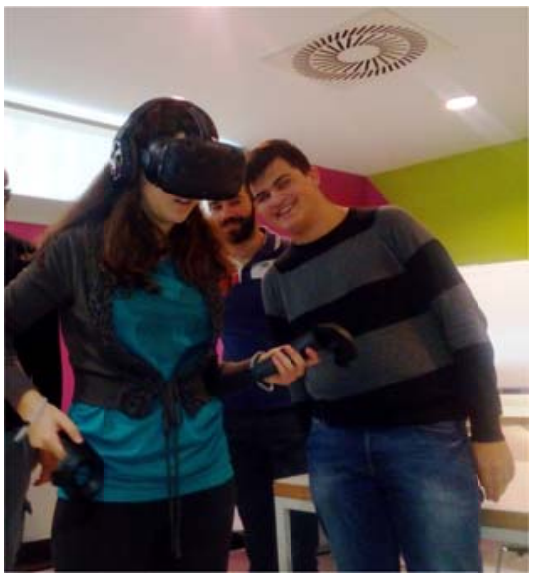
compró en 2014.

\section{Hardware}

El dispositivo de realidad virtual utilizado para NeoTrie VR es el HTC Vive (https://www.vive.com), que actualmente es el que proporciona las mejores prestaciones del mercado. Cuenta con dos “estaciones base”, que

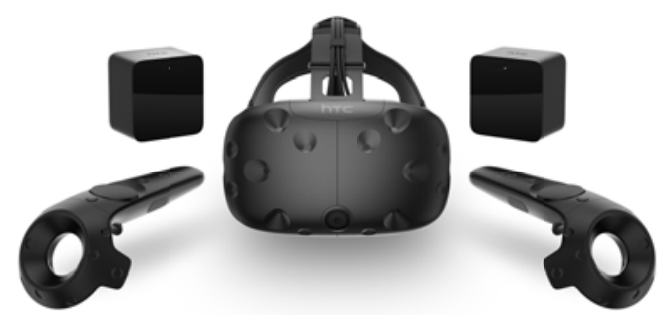
mediante rayos infrarrojos, detectan $\mathrm{y}$ determinan en tiempo real la posición y orientación del casco (y por tanto, del jugador) así como de dos mandos, con los que el usuario puede interactuar con el mundo virtual. Los requisitos mínimos de hardware son los requeridos por el casco HTC Vive: principalmente, una tarjeta gráfica Nvidia GTX 970 o superior, RAM 8 GB, y procesador I5-4600 o superior. 


\section{Unity}

NeoTrie VR está diseñado con el programa Unity ([He], descargable en https://unity3d.com), uno de los más extendidos para desarrollo de videojuegos y entornos 3D. Está basado en $\mathrm{C}++$, y se programa en C\# y en .Net de Microsoft. Unity se lanzó en la conferencia mundial de desarrolladores de Apple en 2005. Desde entonces ha ido creciendo e incorporando nuevas funciones para adaptarse tanto a grandes empresas de desarrollo como a pequeños desarrolladores. Cabe destacar, que juegos que han tenido gran profusión recientemente, como Pokemon Go, han sido desarrollados con Unity.

\section{Modos básicos de NeoTrie VR}

Como hemos indicado al principio, nuestro software permite generar figuras geométricas formadas por vértices, aristas y caras, a tiempo real y en tiempo de compilación, de forma sencilla e intuitiva, sin necesidad de poseer ningún conocimiento de diseño gráfico en 3D.

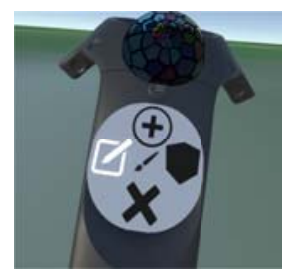

A continuación describimos los modos de uso de los mandos que maneja el jugador para interactuar con el mundo virtual de NeoTrie VR:

1. Modo crear: marcado en los mandos con un signo “más”, permite la creación de vértices al pulsar el gatillo posterior del mando. Las aristas se generan al pulsar dos vértices, y las caras al pulsar dos aristas.

2. Modo edición: marcado con el símbolo de un lápiz, posibilita el desplazamiento de cualquier vértice que hayamos creado por el espacio, además de facilitarnos manipular las distintas proyecciones de los objetos 3d sobre el plano, es decir sus sombras.

3. Modo borrar: permite eliminar los vértices y aristas que hayamos creado.

4. Modo objeto: simbolizado con un cubo, permite desplazar en tres direcciones, cualquier objeto conectado por aristas.

5. Modo anotación o dibujo libre: marcado con un pincel, en el centro del menú, habilita la creación de trazas curvas en realidad virtual.

\section{Actividades y retos con NeoTrie VR}

Las posibilidades didácticas de este juego nos parecen prometedoras y pretendemos profundizar sobre ellas próximamente. Creemos que constituye un recurso muy 
interesante con la que se podrán alcanzar los objetivos y cubrir los contenidos descritos al principio del artículo de una manera efectiva.

El primer contacto que tiene el usuario al entrar en el escenario virtual y comenzar a construir es fascinante. Rápidamente, se da cuenta que puede crear de forma "mágica” en el aire, figuras como nunca antes había imaginado. Puede crear con facilidad estructuras gigantes e introducirse dentro de ellas, como en la cúpula o la red cristalina mostradas a continuación:

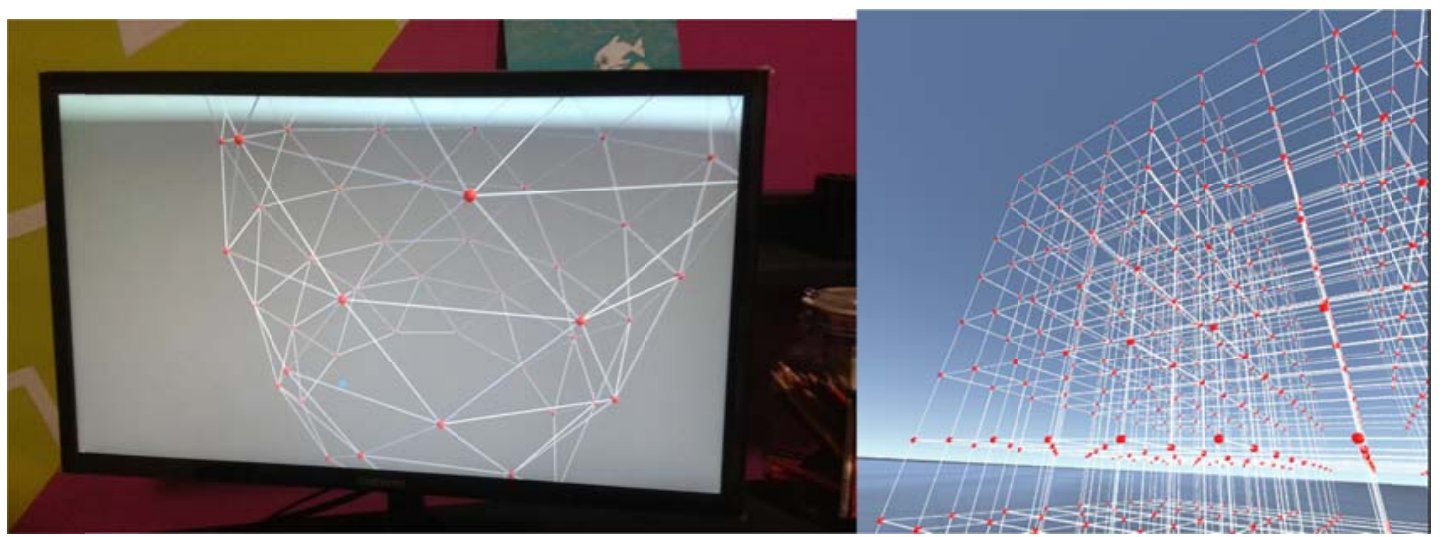

Complementando a la parte manipulativa en el aula, que puede ser la construcción física de figuras geométricas con cartulina o Polifieltros 3d (http://www.polifieltros3d.com, tal y como recomendamos en [PS]), los alumnos pueden construir modelos de cualquier figura en el entorno virtual. Y no solo eso, además pueden modificarlas moviendo los vértices, estirarlas, unir diferentes piezas, solaparlas, etc. utilizando los modos indicados anteriormente.

Por otro lado, NeoTrie VR dispone de una librería de figuras, en las que se incluyen los 5 cuerpos platónicos, los poliedros arquimedianos y muchas otras (importadas del Mathematica), que pueden instanciarse fácilmente con el mando o, más cómodamente, a través de reconocimiento de voz. El profesor puede o bien pedir al alumnado que dibuje dichos cuerpos o llamarlos por voz antes para su posterior reproducción con los mandos. 


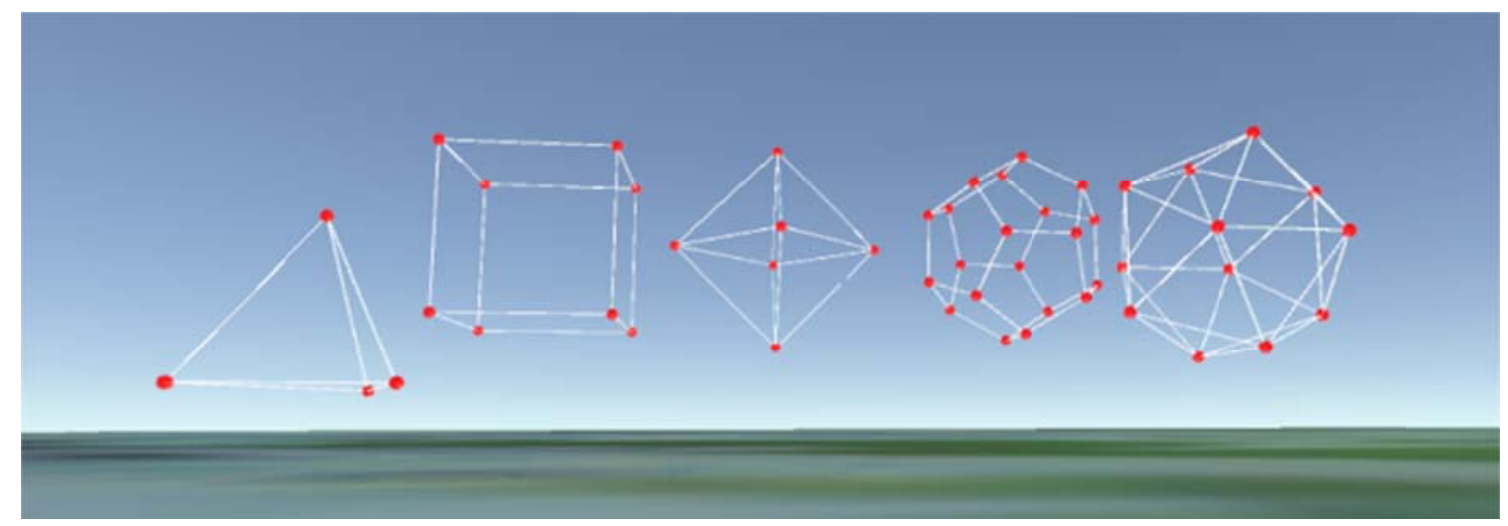

Cuerpos platónicos generados con reconocimiento de voz

El escenario contiene también un foco de luz dinámico, que nos permite ver diferentes sombras de un mismo objeto al mover el foco a conveniencia.

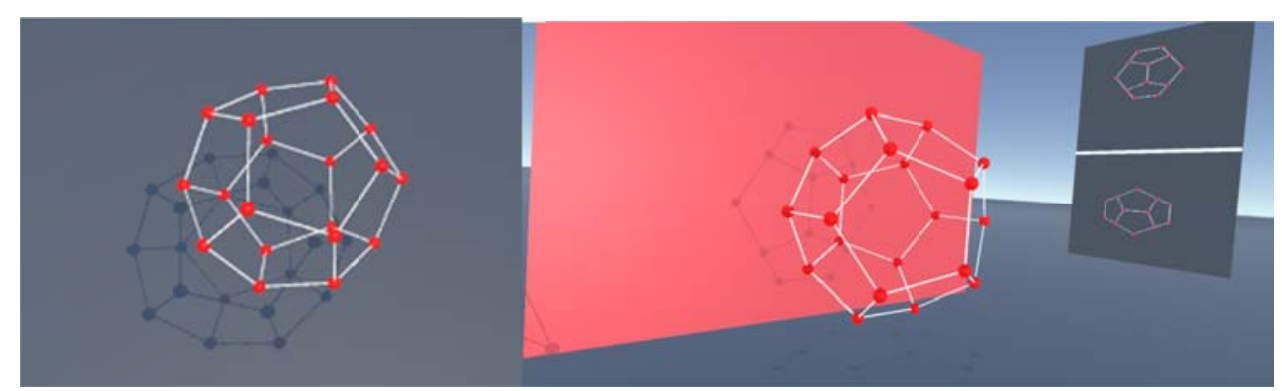

Sombra de un dodecaedro y proyecciones en sistema

$$
\text { diédrico }
$$

Otro reto interesante, es construir una figura en modo libre sin interrupciones y sin sobreescribir las mismas aristas. Las figuras (grafos) que cumplen esta propiedad son los grafos Eulerianos.

El trazado libre es muy útil. Podemos demandar por ejemplo al alumno que reproduzca el trazo de un nudo de

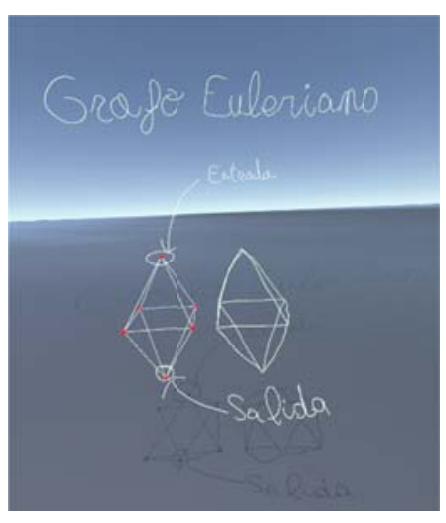
trébol o del nudo figura ocho.

Así, la realidad virtual pone a nuestro alcance una "Pizarra" tri-dimensional, donde podemos dibujar a nuestro alrededor lo que queramos y los matemáticos aprovechamos esta opción para ver en 3D las figuras que más nos interese en cada momento. 


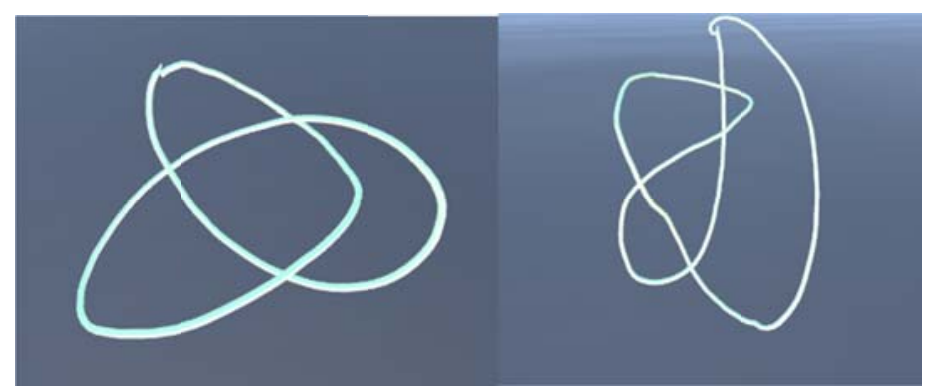

Otra actividad interesante es construir superficies poliédricas y calcular su característica de Euler.

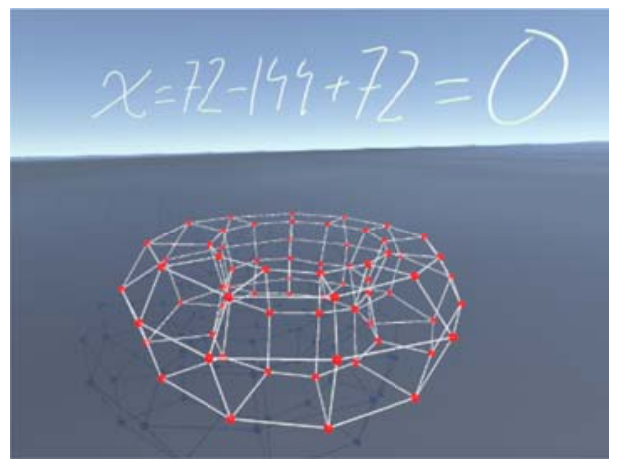

Triangulación de un toro con 72 trapecios

Éstas son sólo una pequeña muestra de actividades que se pueden proponer en el aula. A partir de aquí, el profesorado podrá proponer nuevas ideas o adaptar algunas de las actividades que normalmente realiza en el aula al escenario de NeoTrie VR y compartirlas con otros usuarios online.

\section{Mejoras técnicas futuras}

En un futuro próximo, tenemos previsto implementar la modalidad de multijugador, que permitirá trabajar en el mismo escenario a varios alumnos, incluso de distintos centros o países, para por ejemplo construir un objeto gigante, tipo fractal, o competir para superar retos de problemas en 3D. Además del modo multijugador, podremos guardar las figuras creadas, así como la secuencias para obtenerlas y sus explicaciones, para compartirlas online. Tenemos previsto también incorporar un exportador a formato .STL, lo cual permitirá que cualquier figura creada con nuestro software pueda ser posteriormente tratada con un software de diseño 3D, como por ejemplo Blender ([Ho], http://www.blender.org), o bien su traspaso a una impresora 3d, consiguiendo así, romper el tópico según el cual que para usar una impresora $3 \mathbf{D}$, se necesitan conocimientos previos de diseño gráfico.

En el caso de la parte manipulativa del software, queremos utilizar el dispositivo Leap Motion (http://www.leapmotion.com) que es más intuitivo y permite al usuario manejar 
las figuras virtuales con sus propias manos. Este sensor lo utilizamos ya con unas gafas Oculus Rift para el manejo de superficies topológicas (véase video en [PS]).

\section{Conclusiones}

La realidad virtual nos permite crear un mundo flexible alejado de las limitaciones del mundo físico y más cercano al regido solo por las leyes matemáticas. Esto nos abre las puertas a una pluralidad de posibilidades todavía por descubrir.

Las generaciones futuras tendrán incorporadas la realidad virtual (y variantes, como la realidad aumentada o mixta) como una potente herramienta de trabajo en las aulas.

Sin duda, la realidad virtual proporcionará grandes beneficios en el aprendizaje de las matemáticas, y en especial de la geometría 3D.

Es realmente fascinante observar a niños (y adultos) disfrutar, jugar y crear figuras, moviéndose fácilmente como peces en el agua, dentro de un espacio virtual, amplio y sin limitaciones físicas. Un mundo virtual, o porqué no llamarlo, mágico, donde la imaginación no tiene límite.

\section{Agradecimientos:}

Este proyecto cuenta con el apoyo económico de la Red Española de Topología, del Vicerrectorado de Extensión Universitaria y Deportes, y del Secretariado de Innovación y Docencia Virtual de la Universidad de Almería.

\section{Referencias}

[He] Hess, R. (2011). Blender: Diseño y creatividad. Madrid. Anaya.

[Ho] Hocking, J. (2015). Unity in Action: Multiplatform Game Development in C\# with Unity 5, Manning Publications, Co.

[O] La Voz de Almería (2017). "La realidad virtual se abre paso en los colegios" http://www.lavozdealmeria.es/Noticias/127091/2/La-realidad-virtual-se-abre-paso-enlos-colegios, Artículo de prensa consultado el 14/04/2017.

[PS] Universidad de Almería (2016-17). Proyecto educativo "Let's play to classify surfaces!” https://sites.google.com/a/ual.es/surfaces/materials/NeoTrie-project. Consultado el 21/04/2017 . 